\title{
Modelling for the robust design of layered resonators for ultrasonic particle manipulation
}

\author{
Martyn Hill ${ }^{\mathrm{a}, *}$, Rosemary J. Townsend ${ }^{\mathrm{a}}$, Nicholas R. Harris ${ }^{\mathrm{b}}$ \\ a School of Engineering Sciences, University of Southampton, SO17 1BJ, UK \\ ${ }^{\mathrm{b}}$ School of Electronics and Computer Science, University of Southampton, SO17 1BJ, UK
}

\section{A R T I C L E I N F O}

\section{Article history:}

Received 11 June 2007

Received in revised form 3 June 2008

Accepted 3 June 2008

Available online 17 June 2008

\section{Keywords:}

Acoustic radiation force

Layered resonators

Robust design

Particle manipulation

\begin{abstract}
A B S T R A C T
Several approaches have been described for the manipulation of particles within an ultrasonic field. Of those based on standing waves, devices in which the critical dimension of the resonant chamber is less than a wavelength are particularly well suited to microfluidic, or "lab on a chip" applications. These might include pre-processing or fractionation of samples prior to analysis, formation of monolayers for cell interaction studies, or the enhancement of biosensor detection capability.

The small size of microfluidic resonators typically places tight tolerances on the positioning of the acoustic node, and such systems are required to have high transduction efficiencies, for reasons of power availability and temperature stability. Further, the expense of many microfabrication methods precludes an iterative experimental approach to their development. Hence, the ability to design sub-wavelength resonators that are efficient, robust and have the appropriate acoustic energy distribution is extremely important.

This paper discusses one-dimensional modelling used in the design of ultrasonic resonators for particle manipulation and gives example of their uses to predict and explain resonator behaviour. Particular difficulties in designing quarter wave systems are highlighted, and modelling is used to explain observed trends and predict performance of such resonators, including their performance with different coupling layer materials.
\end{abstract}

(c) 2008 Elsevier B.V. All rights reserved.

\section{Introduction}

Ultrasonic standing waves (USWs) can be used to trap and manipulate particles, and are particularly well suited for the manipulation of micron-scale biological particles in devices of a microfluidic scale [1]. Several different approaches have been employed for the manipulation of particles using ultrasonic fields. For example, focussed ultrasound [2,3] or near-field effects [4] can be used to trap particles prior to analysis, particles can be moved by using two or more opposing transducers to modulate the standing wave field $[5,6]$, or particles can be held and moved within USWs excited by plate waves coupled into the containing fluid $[7,8]$. However, the use of a simple planar layered resonator with a single transducer [9-11] offers the simplest approach to establishing a USW suitable for particle movement.

Planar USW systems may employ resonators that are larger than a wavelength and contain multiple pressure nodal planes $[12,13]$, but for microfluidic scale devices, a resonant cavity with an axial dimension that is lower than the operating wavelength

\footnotetext{
* Corresponding author. Tel.: +44 2380593075 .

E-mail address: m.hill@soton.ac.uk (M. Hill).
}

may be employed [14-16]. Such sub-wavelength resonators typically rely for their operation on precise positioning of the pressure node, to which particles will migrate. In these systems the ability to design a resonator that will operate with a good efficiency and have the required acoustic mode shapes is critical.

A variety of approaches to modelling resonators have been described (see for example $[9,17,18]$ ). This paper uses one-dimensional models implementing impedance transfer relationships $[11,19,20]$.

\section{Layered resonators for particle manipulation}

The structure of a typical planar resonator is shown in Fig. 1 and consists of a transducer which is, in general, bonded to a coupling layer (also known as a carrier layer or matching layer) that serves to isolate the adjacent fluid layer from the transducer. A standing wave is established in the fluid layer by a solid reflector layer.

Particles within the standing wave experience a force, which in most cases of interest acts towards a pressure node of the standing wave. The force on a particle of radius $a$ at position $x$ within the standing wave can be expressed as a function of the spatial gradients of the time averaged kinetic $\left(E_{\mathrm{kin}}(x)\right)$ and potential $\left(E_{\mathrm{pot}}(x)\right)$ energies [21] 
$F(x)=\frac{\partial}{\partial x}\left(\frac{4 \pi a^{3}}{3}\left(\frac{3\left(\rho_{\mathrm{p}}-\rho_{\mathrm{f}}\right)}{\left(2 \rho_{\mathrm{p}}+\rho_{\mathrm{f}}\right)} E_{\mathrm{kin}}(x)-\left(1-\frac{\beta_{\mathrm{p}}}{\beta_{\mathrm{f}}}\right) E_{\mathrm{pot}}(x)\right)\right)$

where $\beta$ and $\rho$ are the compressibility and the mass density of the fluid and the particle, indicated by subscripts $f$ and $p$ respectively. The wave number, $k$ is equal to $2 \pi / \lambda$ where $\lambda$ is the wavelength of the standing wave.

The problem of modelling particle behaviour in the standing wave then becomes one of describing the variation of acoustic parameters through the acoustic field such that the energy parameters $E_{\mathrm{kin}}(x)$ and $E_{\mathrm{pot}}(x)$ can be calculated. The rest of the paper discusses the characterisation of the field itself in order to provide the behaviour required for the correct operation of the device (chiefly nodal position and energy density).

\section{Simple two-layer model}

\subsection{Background theory}

An approach to the characterisation of the underlying properties of resonators is to begin by looking at the modal solutions of a two-layer system consisting of a fluid layer with a rigid boundary coupled with a reflector layer with a pressure-release boundary [20]. Such a model is not, in itself, sufficient to understand the behaviour of a resonator such as that shown in Fig. 1. However, it can act as a starting point, suggesting layer properties able to provide the required nodal behaviour. Additional layers can then be added and their parameters adjusted in a fuller model to maintain, or fine-tune resonator response. The two-layer approach simplifies to solving

$k_{\mathrm{f}} t_{\mathrm{f}}=\tan ^{-1}\left(\frac{r_{\mathrm{f}}\left(r_{\mathrm{r}}^{2}+r_{0}^{2} \tan ^{2}\left(k_{\mathrm{r}} t_{\mathrm{r}}\right)\right)}{r_{\mathrm{r}} \tan \left(k_{\mathrm{r}} t_{\mathrm{r}}\right)\left(r_{\mathrm{r}}^{2}-r_{0}^{2}\right)}\right)$

where $t_{\mathrm{r}}$ and $t_{\mathrm{f}}$ are the thicknesses of the reflector and fluid layers respectively, $k$ is the wave number and $r_{\mathrm{f}}$ and $r_{0}$ are the acoustic impedances of the fluid and reflector layers, respectively. A given ratio of the thicknesses of the two layers (i.e. a fixed design) is represented by a straight line passing through 0,0 on the graph in Fig. 2 , which shows the first four solutions of Eq. (2), plotted as the ratio of thickness to wavelength of the fluid layer to the ratio of thickness to wavelength of the reflector layer.

A fluid layer having two perfectly rigid boundaries would have modal solutions corresponding to horizontal lines at $t_{\mathrm{f}} / \lambda_{\mathrm{f}}=0.5,1$, etc. where $\lambda_{\mathrm{f}}$ is the ultrasonic wavelength in the fluid layer. Similarly, a reflector bounded by two pressure-release surfaces would show half wave resonances as vertical lines corresponding to $t_{\mathrm{r}} / \lambda_{\mathrm{r}}=0.5,1$, etc. There is, however, no modal solution at $(0.5$, 0.5 ) on the graph, but region $C$ shows how the modes of the cou-

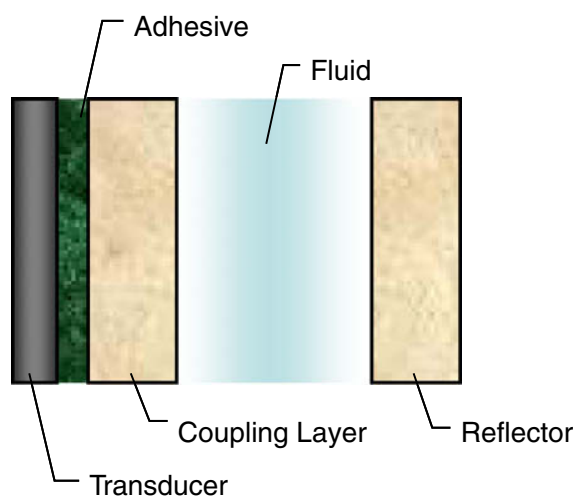

Fig. 1. Typical structure of a planar layered resonator.

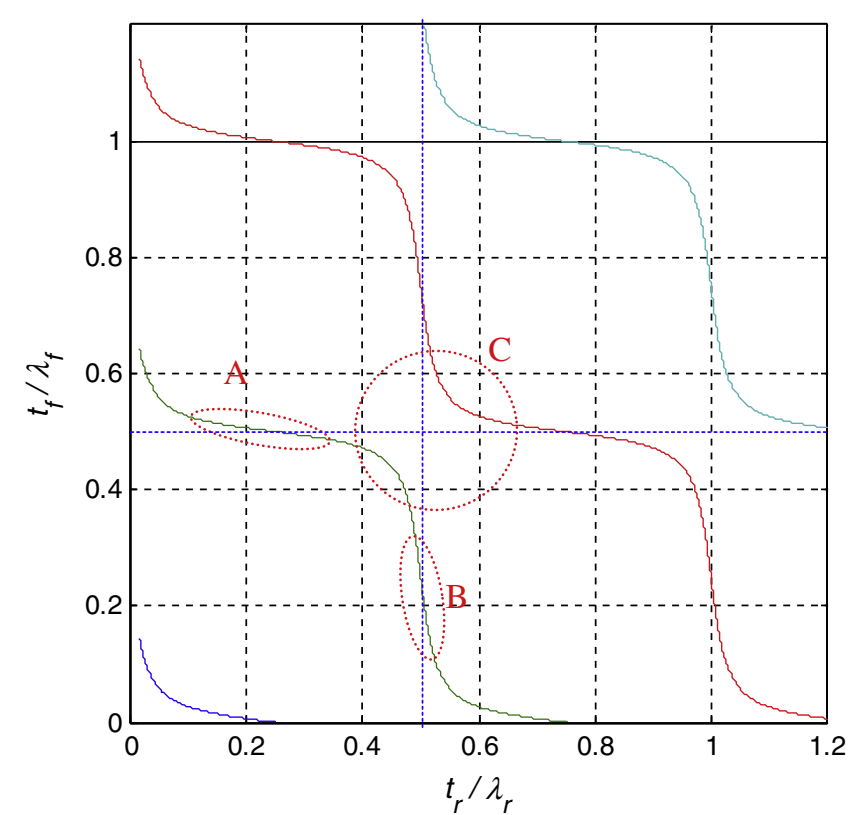

Fig. 2. The first four solutions of Eq. (2), based on [20]. Parameters $t_{\mathrm{r}}$ and $t_{\mathrm{f}}$ are the thicknesses of the reflector and fluid layers, respectively and $\lambda_{\mathrm{r}}$ and $\lambda_{\mathrm{f}}$ are ultrasonic wavelengths in those layers.

pled system split when adjacent layers would have coincident resonances if isolated. The parameter in Fig. 2 that indicates the position of the pressure node within the fluid layer is $t_{\mathrm{f}} / \lambda_{\mathrm{f}}$. In order to place a node centrally, in this two-layer model, a reflector thickness of a quarter-wavelength is required, i.e. $t_{\mathrm{r}} / \lambda_{\mathrm{r}}=0.25$, as shown in area $A$ of Fig. 2. It can also be seen from area $A$ that the solid line representing the solution to Eq. (2) is relatively flat in that region, indicating that the position of the node should be stable with regards to the reflector layer thickness. Even with the combination of a half wavelength fluid layer and a quarter-wavelength reflector layer, the influences of the coupling layer and transducer need to be considered, and Fig. 3a shows how the frequency of maximum energy density in the fluid layer, and hence the position of the node within the fluid layer, varies as a function of coupling layer thickness. With the parameters used in this system (taken from [11]) the variation in frequency is relatively small, and selection of a quarter-wavelength coupling gives the most stable frequency, but a relatively low value of peak energy. The material used for the coupling layer is investigated in more detail later.

The highest values of energy density occur either side of the points at which the coupling layer equals half wavelength multiples, but with a significant dip for the half wavelength coupling layer itself. This dip, however, becomes less pronounced at lower $Q$ factors. If the $Q$ factors are reduced from 200 and 500 for the coupling and fluid layers, respectively (Fig. 3a) to 50 for both (Fig. 3b), the frequency variation of the peak remains similar, but the peak energy density characteristics change significantly. These $Q$ factors seem low, but their values represent observed losses throughout the resonant device and will in general be much lower than material $Q$ factors [9]. A similar observation is made later for quarterwavelength fluid layer systems where coupling layer materials have been investigated.

\section{Modelling of quarter-wavelength devices}

The need for robust design of resonators becomes particularly important when dealing with quarter-wavelength devices. A quarter-wavelength resonance is observed when the fluid layer is at a quarter-wavelength and the reflector layer is a half wavelength 
a
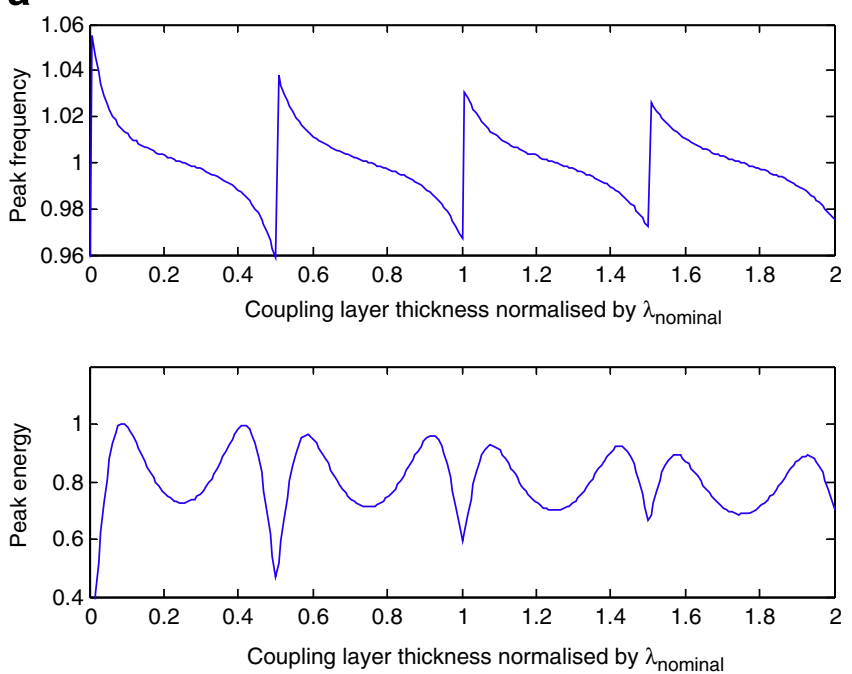

b
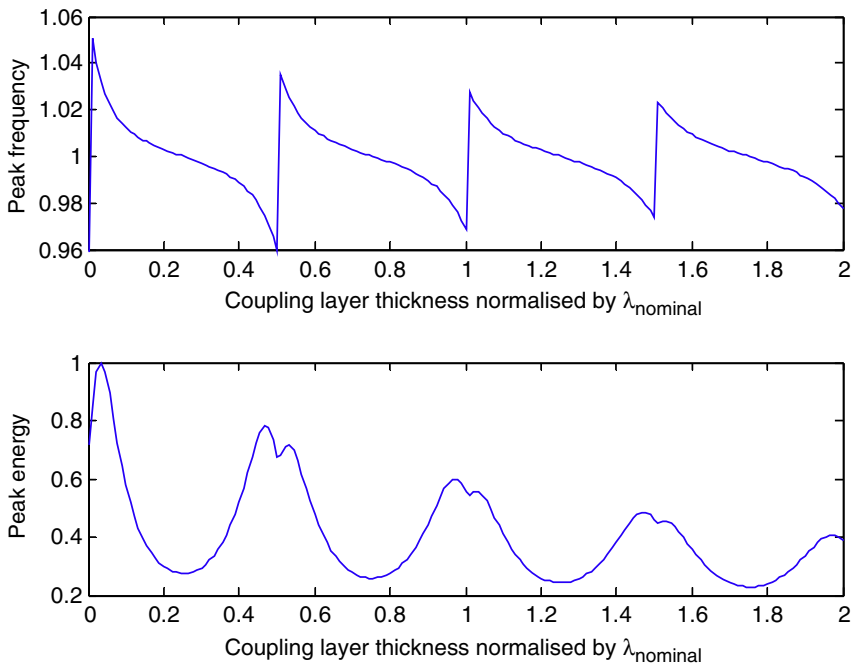

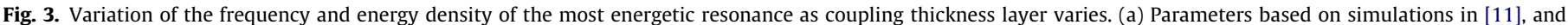
normalised against the nominal resonance frequency. (b) With lower $Q$ factors in coupling and fluid layers.

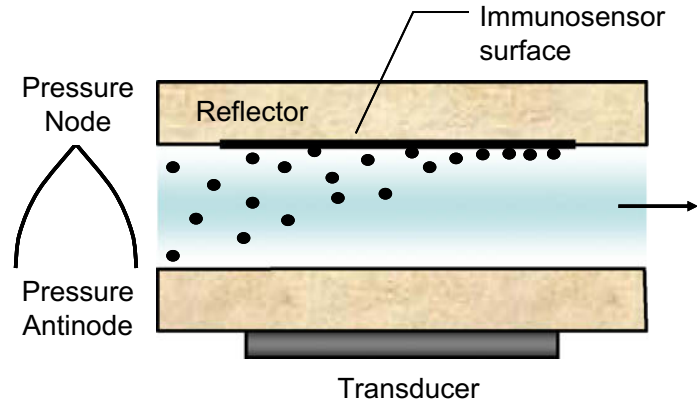

Fig. 4. Schematic representation of a quarter-wavelength resonator forcing particles up against a solid surface with an immunosensor coating.

thick, in the region marked $B$ in Fig. 2. Such a system may be used to force particles against, or close to, a solid surface as shown in Fig. 4 which is designed to enhance particle capture on an immunosensor surface.

A simple simulation suggests that for fixed values of coupling layer and reflector thickness, simply varying the fluid layer thickness should enable the pressure node to be positioned at, or close to, the reflector layer. This can be seen from the simulations of a silicon/Pyrex resonator shown in Fig. 5, which use the parameters shown in Table 1.

However the gradient of the solution line in region $B$ of Fig. 2 suggests that the nodal position is likely to be extremely sensitive to reflector layer thickness.

Martin et al. [22] investigated the acoustics of such a system with the aim of forcing spores onto an antibody coated surface using a $3 \mathrm{MHz}$ USW. The device was tested in batch and flowthrough modes and it was found that the efficacy of capture was critically dependent on the reflector layer thickness. When a $980 \mu \mathrm{m}$ thick reflector was used, there was almost no capture of the BG spores. Capture increased with a $1000 \mu \mathrm{m}$ reflector, peaked with a thickness of about $1100 \mu \mathrm{m}$ and fell away significantly with a reflector thickness of $1300 \mu \mathrm{m}$. This was explained by 1D simulations of the acoustic pressure for different reflector thicknesses, as shown in Fig. 6a. With a reflector thickness of $980 \mu \mathrm{m}$, the pressure node is in the fluid, away from the reflector boundary, so particles are forced away from the antigen surface. With a $1000 \mu \mathrm{m}$ reflec-
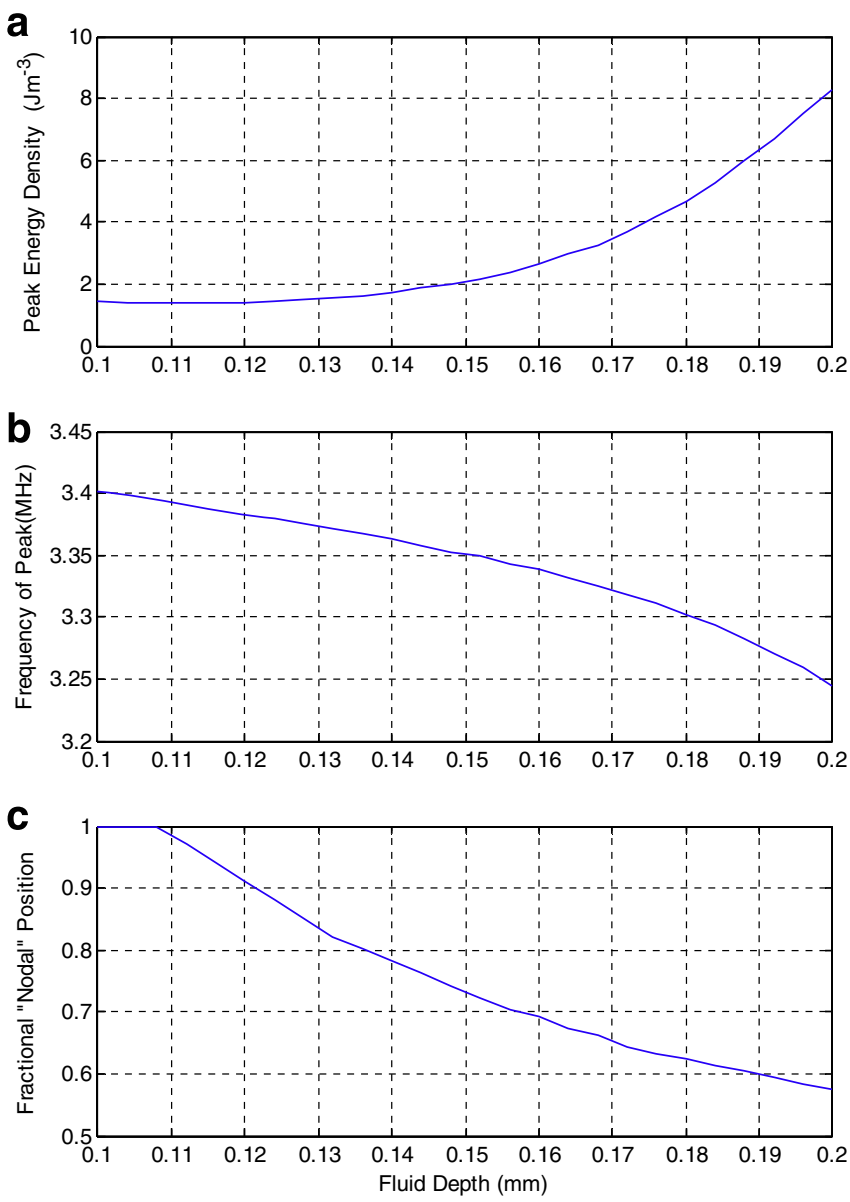

Fig. 5. Simulated values of peak energy density, peak frequency and nodal position for silicon microfabricated quarter-wavelength resonator.

tor, the node is just in the reflector, so particles will be forced to the surface. A $1200 \mu \mathrm{m}$ reflector places the node well into the reflector, but also brings a pressure antinode into the fluid, causing many particles to be forced to the opposite boundary. Hence there is an optimum positioning of the node that is dependent on the 
Table 1

Quarter wave simulation parameters

\begin{tabular}{llll}
\hline & Coupling & Fluid & Reflector \\
\hline Thickness $(\mathrm{m})$ & $5.25 \mathrm{e}-4$ & Varying & $1.60 \mathrm{e}-3$ \\
Density $\left(\mathrm{kg} \mathrm{m}^{-3}\right)$ & $2.34 \mathrm{e}+3$ & $1.00 \mathrm{e}+3$ & $2.20 \mathrm{e}+3$ \\
Speed of sound $\left(\mathrm{m} \mathrm{s}^{-1}\right)$ & $8.43 \mathrm{e}+3$ & $1.50 \mathrm{e}+3$ & $5.43 \mathrm{e}+3$
\end{tabular}

a
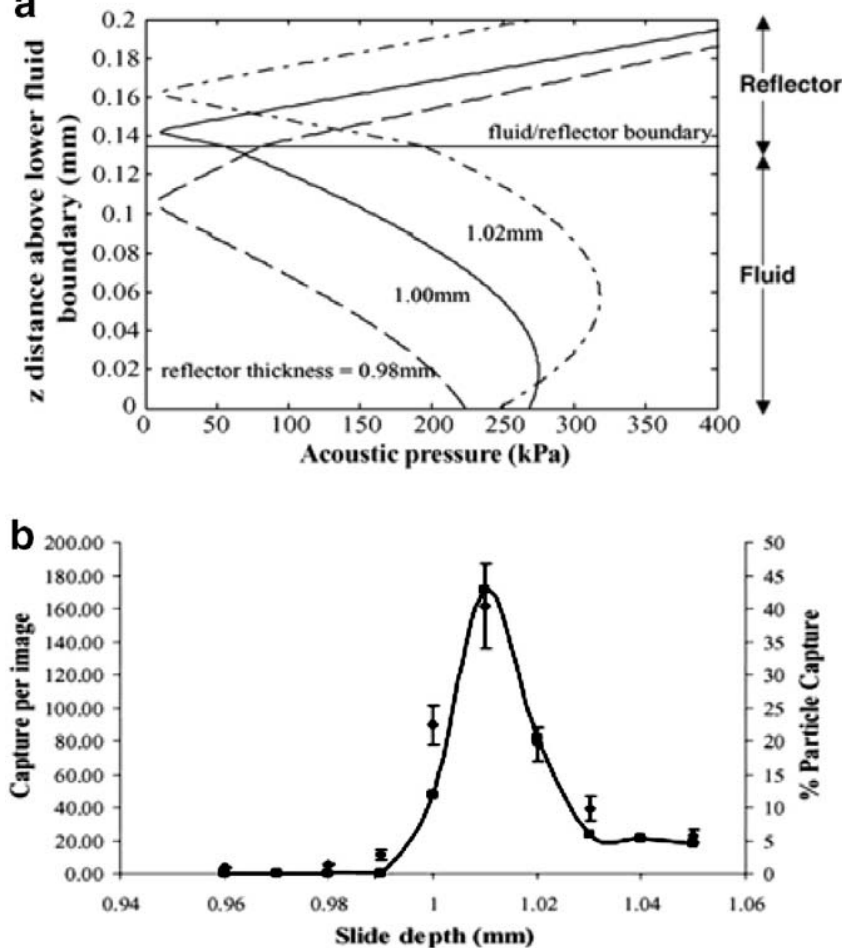

Fig. 6. Acoustic simulation of the pressure profile across the chamber used by Martin et al. [22] for different reflector thicknesses (a), and simulations of particle capture (line) compared with experimental data (b). Reproduced from [22] with permission from Elsevier.

reflector thickness and a significant decrease in capture efficiency on each side of this thickness. When flow and particle tracking were added to the acoustic model [23] it was possible to predict the nature of the dependence of particle capture on reflector depth, as shown in Fig. $6 \mathrm{~b}$.

\section{Selecting operating points from 2D plots}

There are other applications in which it is required to place a pressure node close to, but not on, a boundary with a solid layer. Such a "near quarter-wave" resonator has been designed for con-

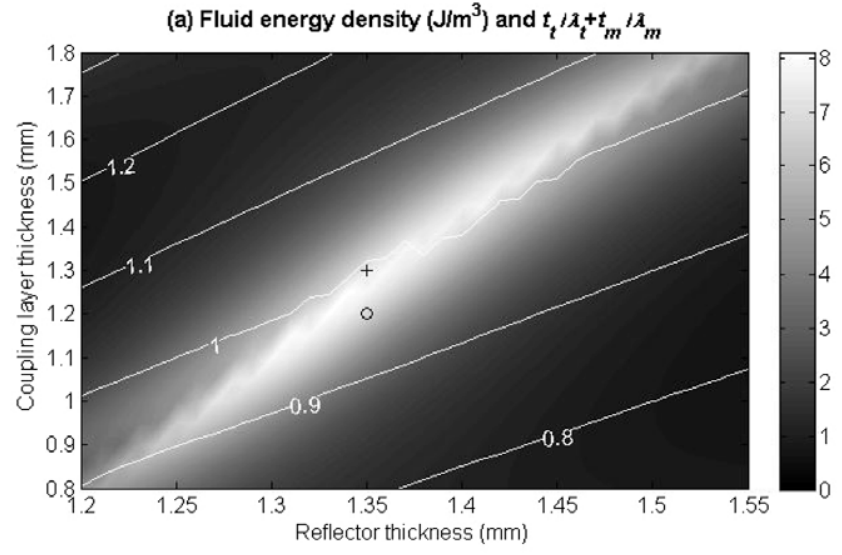

(b) Location of pressure minimum $\left(x h_{f}\right)$ and $t_{t} / \lambda_{t}+t_{m} / \lambda_{m}$

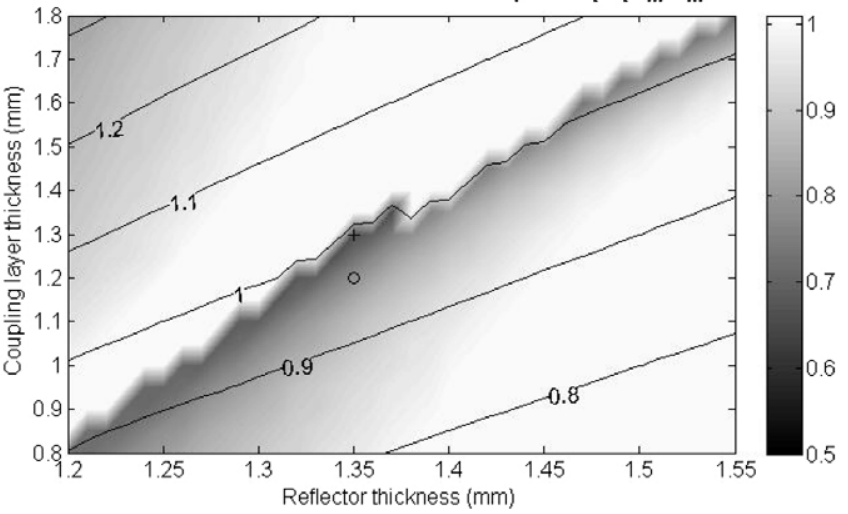

Fig. 8. Acoustic energy density in the fluid layer (upper) and fractional position of pressure minimum (lower) with contours representing the coupled transducer and coupling layer thickness in wavelengths.

centrating particles prior to analysis [24] and is shown schematically in Fig. 7.

In this case the aim was to move particles to within $20 \mu \mathrm{m}$ of the reflector layer in a $180 \mu \mathrm{m}$ cavity. In order to achieve this, multiple simulations were completed to predict the sensitivity of the energy density and nodal position to relevant geometric parameters. An example is shown in Fig. 8 in which these parameters are plotted against reflector and coupling layer thickness. For each geometric design, the acoustic energy within the fluid layer is determined over a small range of frequencies in order to isolate the fluid quarter-wavelength mode. Acoustic energy density and corresponding position of the pressure minimum are recorded for the frequency where energy density is seen to peak.

The parameters used in the simulations are shown in Table 2, with the values of $Q$ factor inferred by matching modelled and experimentally derived electrical input impedance spectra for:

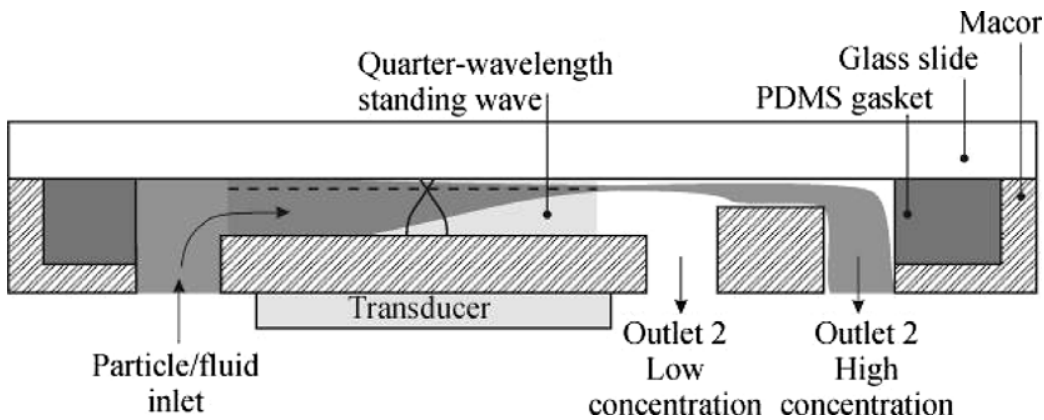

Fig. 7. Schematic representation of a "near quarter-wave" concentrator. 
Table 2

Concentrator simulation parameters

\begin{tabular}{lllll}
\hline Layer & $\begin{array}{l}\text { Thickness } \\
(\mu \mathrm{m})\end{array}$ & $\begin{array}{l}\text { Density } \\
\left(\mathrm{kg} / \mathrm{m}^{3}\right)\end{array}$ & $\begin{array}{l}\text { Sonic velocity } \\
(\mathrm{m} / \mathrm{s})\end{array}$ & $\begin{array}{l}Q \\
\text { factor }\end{array}$ \\
\hline Glue & 10 & 1080 & 2640 & 2 \\
Macor coupling & $800-1800$ & 2540 & 5510 & 100 \\
Fluid & 180 & 1000 & 1480 & 50 \\
Reflector & $1200-1550$ & 2470 & 5600 & 100 \\
\hline
\end{tabular}

1. the isolated transducer,

2. the transducer, glue and coupling layers,

3. the full system.

In Fig. 8 the white diagonal bar across plot (a) suggests that both reflector and coupling layer thicknesses are important parameters to consider when designing to maximise acoustic energy density and therefore radiation force. Similarly, plot (b) indicates how the design influences the position of the pressure minimum within the fluid layer and how the thicknesses chosen can result in the pressure node moving from within the fluid layer a small distance from the reflector layer (grey) and into the reflector such that particles are pushed up to the reflector surface (white). The irregularity of the contour representing a single wavelength is due to a coincidence of the coupled transducer/matching layer resonance and the half wavelength resonance of the reflector. Pressure amplitude plots for the two points ('o' $t_{\mathrm{r}}=1350 \mu \mathrm{m}$ and $t_{\mathrm{c}}=1200 \mu \mathrm{m}$, and ' + ' $t_{\mathrm{r}}=1350 \mu \mathrm{m}$ and $t_{\mathrm{c}}=1300 \mu \mathrm{m}$ ) marked in Fig. 8 are shown in Fig. 9.

It can be seen that point '+' corresponds to a higher energy in the coupling layer, although the pressure profile in the fluid layer is similar. However from Fig. 8 point 'o' is far more robust in terms of nodal position, despite having a lower energy.

Just as the damping in the system alters the characteristics between Fig. 3a and b, this system is also sensitive to changes in $Q$ factors. Fig. 10 is a repeat of the simulation shown in Fig. 8 but with a significantly higher reflector $Q$ factor. The nodal position (lower figure in each case) as a function of layer thicknesses remains robust to layer thicknesses and is similar in both figures. Not surprisingly the magnitude of the energy density in the fluid layer (upper figure) changes significantly between the simulations. Further, the form of the energy density surface has changed. In the more highly damped system of Fig. 8, the maximum energy density lies close to the irregular single wavelength contour, while for the higher $Q$ Fig. 10, the maximum energy lies either side of this contour in a manner similar to the coupled resonances described in [11]. Hence

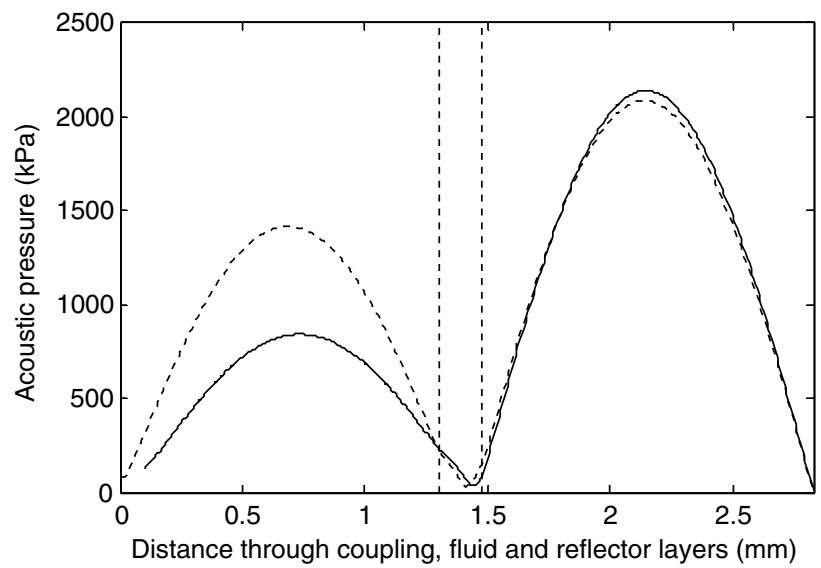

Fig. 9. Acoustic pressure profile for the two potential design points marked in this figure - 'o' (solid) and '+' (dotted).

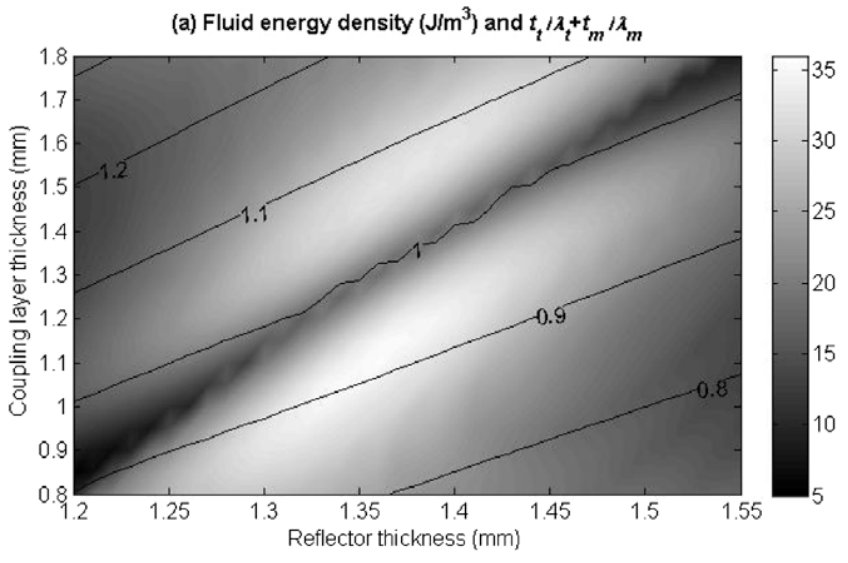

(b) Location of pressure minimum $\left(x \hbar_{f}\right)$ and $t_{t} / \lambda_{t}+t_{m} / \lambda_{m}$

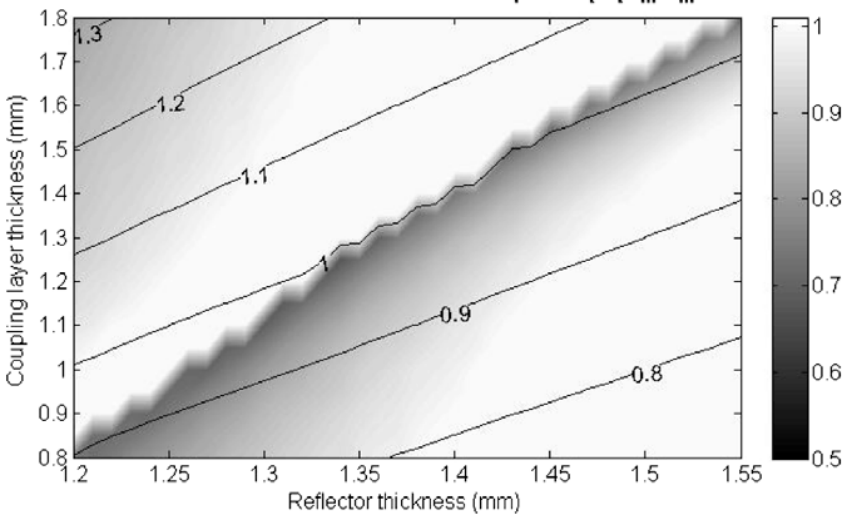

Fig. 10. Acoustic energy density in the fluid layer (upper) and fractional position of pressure minimum (lower) with contours representing the coupled transducer and coupling layer thickness in wavelengths but with higher $Q$ factors in the layers than used in Fig. 9.

the position of the node in these systems appears to be relatively robust to the damping factors.

Fig. 11 shows a prototype quarter wave concentrator in which $t_{\mathrm{r}}=1440 \mu \mathrm{m}$ and $t_{\mathrm{c}}=1040 \mu \mathrm{m}$. This device provided a factor of four concentration with $1 \mu \mathrm{m}$ diameter particles and the nodal position was as predicted by modelling using high $Q$ factors, although the damping and hence energy density characteristics proved to be closer to those shown in Fig. 8 on experimental evaluation.

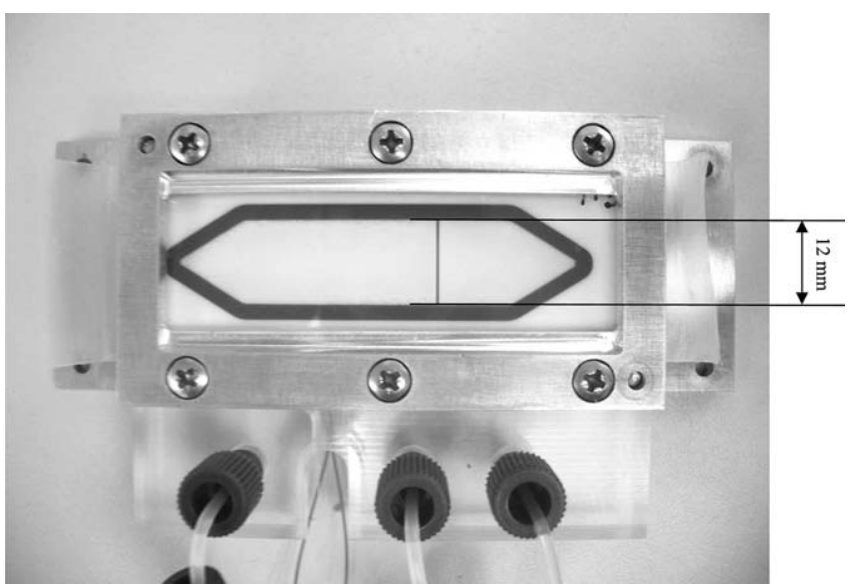

Fig. 11. Prototype quarter-wavelength concentrator. 


\section{Effect of varying the coupling layer material}

As discussed previously, the coupling layer material can have an effect on the nodal positions, and the amount of energy stored within the fluid layer. Practical considerations are also important in designing particle manipulators, such as bio-compatibility, ease of manufacture, and cost, so it is useful to know what range of materials can be used and how they affect the performance. The device shown in Fig. 11 has a coupling layer manufactured from Macor, a machinable ceramic, similar to glass. Devices with coupling layers made from other materials were also constructed. The materials investigated were aluminium and brass. It is known that the coupling layer material influences the acoustic energy density and the maximum radiation force experienced by a particle. This is highly relevant to quarter-wavelength systems as the success of a resonator typically relies on maximising the acoustic energy density. In the case of a quarter wave device, the quarter wave mode is a lot less energetic than a typical half wave device and maximising the efficiency to avoid heating effects in the other layers requires careful design. Quarter wave devices are much less efficient than half wave devices because of the reliance on a reflector layer resonance where much of the acoustic energy is dissipated.

Initial simulations were used to design near quarter-wavelength resonators operating around $2 \mathrm{MHz}$ for each coupling layer material. The acoustic pressure profiles within these devices are similar to that shown in Fig. 9 where a half wavelength resonance is seen in the reflector layer above the fluid chamber. This resonance imposes a node at the fluid/reflector boundary and for certain fluid depths will result in a quarter-wavelength "resonance" in the fluid layer. This mode forces suspended particles up to this surface.

For the experimental devices a transducer with a resonance close to the operating frequency of the assembled chamber was used (Ferroperm PZ26, $1 \mathrm{~mm}$ thick). To give comparable acoustic pressure profiles in the fluid layer, the model was used to select coupling layer and fluid layer thickness dimensions. In each case the fluid layer thickness was chosen to be $0.18 \mathrm{~mm}$ and coupling layer thicknesses of 1.0, 1.4, and 1.2 were selected for brass, aluminium and Macor, respectively. Table 3 contains the measured thickness dimensions and acoustic properties used in the initial modelling.

Although the modelling suggests good results for the pressure profile, the model requires calibrating with experimental data to provide absolute values for the acoustic parameters. This is done by taking acoustic energy density measurements for the different chambers and then adjusting the model parameters such as material $Q$ factors, to get the best match. Acoustic energy density measurements are taken between 1.96 and $2.1 \mathrm{MHz}$ at $10 \mathrm{kHz}$ intervals. These measurements are made by levitating a polystyrene particle and recording the threshold voltage where the particles begin to sediment, similar to the method described by Martin et al. [22]. For the particles, fluid (water) and frequency used, this threshold voltage corresponds to pressure amplitude of $33 \mathrm{kPa}$. During experiments, the position of the node is difficult to measure accurately but is reasonably consistent with predictions of the pressure profile such as that shown in Fig. 9. As the acoustic pressure amplitude is proportional to the transducer voltage, the pressure amplitude $P_{0}$ resulting from a $10 \mathrm{~V}_{\mathrm{pkpk}}$ voltage is recorded and converted to an energy density measurement using (3):

$\langle\bar{\varepsilon}\rangle=\frac{1}{4} P_{0}^{2} \beta_{\mathrm{w}}$

where $\langle\bar{\varepsilon}\rangle$ is the acoustic energy density and $\beta_{\mathrm{w}}$ is the bulk modulus of water, with the results presented in Fig. 12.

These plots show that the acoustic energy density peaks at frequencies where resonant modes are encountered. Predicted energy density is also shown, where the input parameters to the model have been modified to improve the match with both impedance and energy density measurements. Notably the $Q$ factors have been adjusted to 300, 100 and 100 for brass, aluminium and Macor,
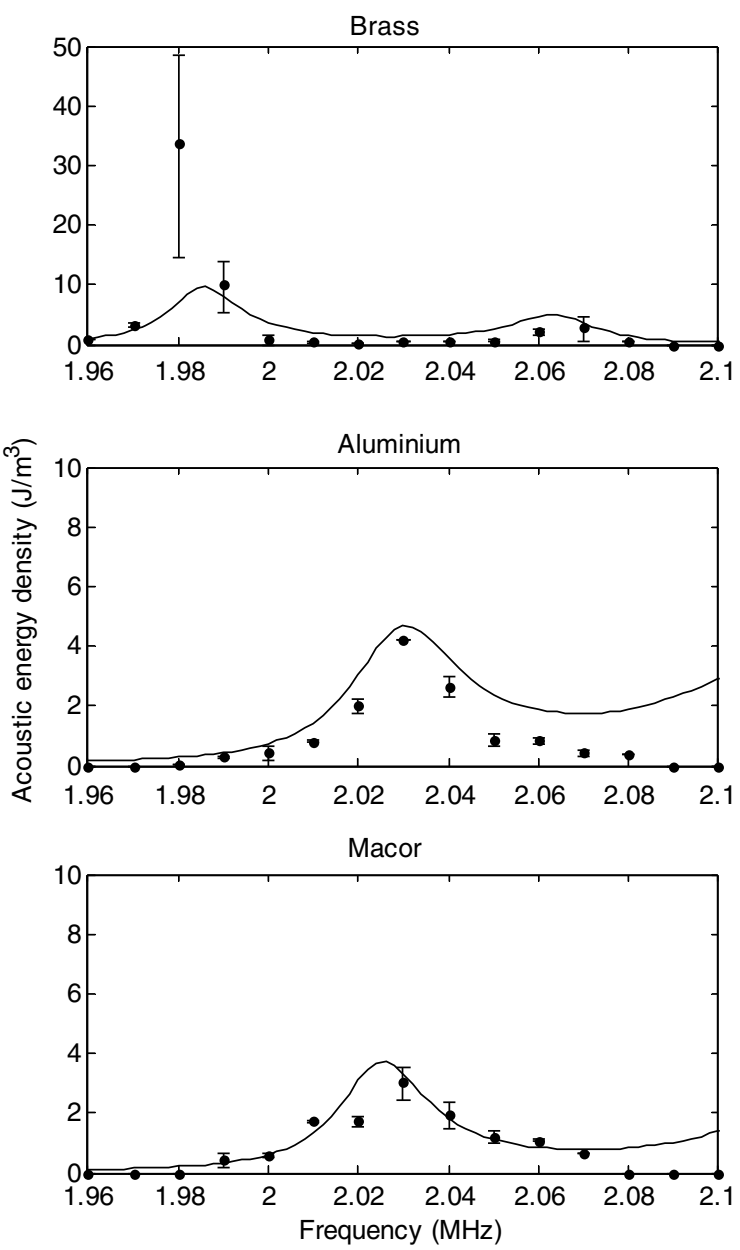

Fig. 12. Comparison between measured (pointes with error bars) and modelled (solid lines) acoustic energy density.

Table 3

Dimensions of experimental samples

\begin{tabular}{|c|c|c|c|c|c|c|c|}
\hline \multirow[t]{2}{*}{ Coupling layer material } & \multicolumn{2}{|c|}{ Coupling layer thickness (mm) } & \multicolumn{2}{|c|}{ Fluid layer thickness (mm) } & \multirow{2}{*}{$\begin{array}{l}\text { Density } \\
\left(\mathrm{kg} / \mathrm{m}^{3}\right)\end{array}$} & \multirow{2}{*}{$\begin{array}{l}\text { Sonic velocity } \\
(\mathrm{m} / \mathrm{s})\end{array}$} & \multirow{2}{*}{$\begin{array}{l}\text { Acoustic impedance } \\
\text { (MRayl) }\end{array}$} \\
\hline & Design & Measured & Design & Measured & & & \\
\hline Brass & 1.00 & 1.08 & 0.18 & 0.17 & 8640 & 4700 & 40.6 \\
\hline Aluminium & 1.40 & 1.42 & 0.18 & 0.175 & 2700 & 6420 & 17.3 \\
\hline Macor & 1.20 & 1.17 & 0.18 & 0.19 & 2540 & 5510 & 14.0 \\
\hline
\end{tabular}

Properties of materials taken from [25]. 
respectively. Similarly, the $Q$ factors in the fluid and reflector are low at $\sim 30$ and $\sim 100$, respectively. In the case of brass, two peaks can be seen although the model suggests that the peak around $1.98 \mathrm{MHz}$ is a combined transducer and coupling layer resonance. This mode appears to be particularly energetic and may be reinforced, for example, by structural modes.

To compare each material more directly, the model was used to simulate the effect of varying coupling layer thickness while applying identical reflector and fluid layer properties. Fig. 13 shows the predicted peak energy density for a range of coupling layer thicknesses as a fraction of wavelength $\left(t_{\mathrm{m}} / \lambda\right)$ and where the transducer voltage is a constant $10 \mathrm{~V}_{\mathrm{pkpk}}$. Note that Fig. 13 is generated by locating the frequency at which the quarter-wavelength mode (reflector resonance) occurs and omits any other resonant modes close to this frequency. It therefore does not include the high energy resonance seen at $1.98 \mathrm{MHz}$ in the experimental results for the brass coupling material.

In general, the energy density is comparable for the materials considered, although the material does have a limited impact in the maximum energy density achievable. Peaks suggest that for all the materials tested a coupling layer thickness of just under $n \cdot t_{\mathrm{m}} / 2 \lambda(n=1,2$ only shown) results in a higher acoustic energy. The trade-off in this case is that the acoustic node moves away from the surface and further into the fluid layer at these dimensions as shown in Fig. 13c. As the transducer is operating close to a half wavelength, the peaks also coincide with a wavelength resonance in the transducer and coupling layer combined where if this structure were isolated from the fluid and reflector would have
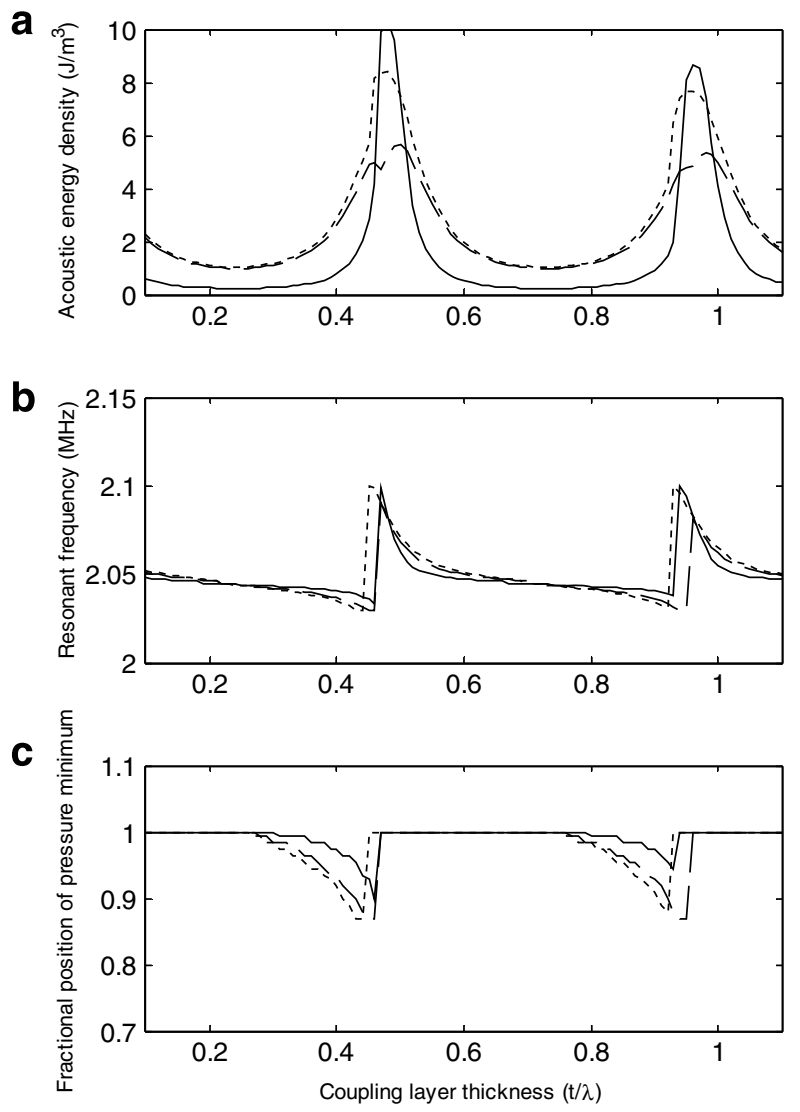

Fig. 13. Predicted peak acoustic energy density in the fluid layer for a range of coupling layer thicknesses for brass (solid), aluminium (dashed) and Macor (dotted). Showing (a) acoustic energy density, (b) the quarter-wavelength excitation frequency and (c) the fractional position of the pressure minimum within the fluid chamber. a pressure node located on the coupling layer surface. The close proximity of this coupled resonance to the quarter-wavelength mode may therefore be responsible for the change in energy density and movement of the node towards the coupling layer surface. Also, for an increase in $n$ the energy density decreases, probably due to greater losses within a progressively thicker coupling layer.

The observations made in Fig. 13 are similar to those in Fig. 3b for a half wavelength system. This suggests that the design of the coupling layer and transducer can be decoupled from the fluid and reflector layer to some extent. For example, the position of the nodal plane(s) within a fluid chamber depends on the controlled design of the fluid and reflector design and aided by Fig. 2. Although the coupling layer and transducer will influence the node position and energy density, they have a small effect relative to fluid/reflector design and considering the wide range of coupling layer/transducer designs which could feasibly drive the system.

\section{Conclusions}

One-dimensional acoustic modelling has helped identify specific parameters which influence the robust design of resonators for particle manipulation. The nodal position and energy density are typically important factors in resonator performance, and the choice of layer dimensions and material properties influence these factors significantly. For example, the careful selection of $Q$ factors for the various materials and layers used to construct these resonators may be used to help relax dimensional tolerances. It appears that the influence of the coupling layer on the performance of both half and quarter-wavelength resonances are related, with the proximity of the coupling layer/transducer mode impacting upon the characteristics of fluid/reflector modes.

It has also been shown that the behaviour of quarter-wavelength modes can be more fully understood based on a comparison of modelled and experimental data. Using experimental data to refine the simulation input parameters it is possible to predict the acoustic energy density to well within the correct order of magnitude. This is important, for example, for bio-sensing applications where the location of the pressure node and strength of the field influences significantly the feasibility of such devices. The choice of material used to couple between the transducer and fluid manipulation chamber determines to a limited extent the maximum energy density achievable, although coupling layer thickness appears to have a greater impact. Therefore a range of materials can be used for the construction of the coupling layer.

While 1D models are able to provide very useful, and in the case of nodal position accurate, predictions of device behaviour, the influences of lateral field variations are also of significance [26]. These variations are the main limitation on the performance of the concentrator described here [24].

\section{Acknowledgements}

The authors gratefully acknowledge the support and funding from the Defence Science and Technology Laboratory (DSTL), Porton Down, UK.

\section{References}

[1] T. Laurell, F. Petersson, A. Nilsson, Chip integrated strategies for acoustic separation and manipulation of cells and particles, Chemical Society Reviews 36 (2007) 492-506.

[2] M. Wiklund, J. Toivonen, M. Tirri, P. Hanninen, H.M. Hertz, Ultrasonic enrichment of microspheres for ultrasensitive biomedical analysis in confocal laser-scanning fluorescence detection, Journal of Applied Physics 96 (2004) 1242-1248. 
[3] H.M. Hertz, Standing-wave acoustic trap for nonintrusive positioning of microparticles, Journal of Applied Physics 78 (1995) 4845-4849.

[4] T. Lilliehorn, U. Simu, M. Nilsson, M. Almqvist, T. Stepinski, T. Laurell, J. Nilsson, S. Johansson, Trapping of microparticles in the near field of an ultrasonic transducer, Ultrasonics 43 (2005) 293-303.

[5] G. Whitworth, M.A. Grundy, W.T. Coakley, Transport and harvesting of suspended particles using modulated ultrasound, Ultrasonics 29 (1991) 439-444.

[6] Y. Abe, M. Kawaji, T. Watanabe, Study on the bubble motion control by ultrasonic wave, Experimental Thermal and Fluid Science 26 (2002) 817-826.

[7] A. Haake, A. Neild, G. Radziwill, J. Dual, Positioning, displacement, and localization of cells using ultrasonic forces, Biotechnology and Bioengineering 92 (2005) 8-14.

[8] A. Neild, S. Oberti, F. Beyeler, J. Dual, B.J. Nelson, A micro-particle positioning technique combining an ultrasonic manipulator and a microgripper, Journal of Micromechanics and Microengineering 16 (2006) 1562-1570.

[9] M. Gröschl, Ultrasonic separation of suspended particles - Part I: Fundamentals, Acustica 84 (1998) 432-447.

[10] J.J. Hawkes, W.T. Coakley, Force field particle filter, combining ultrasound standing waves and laminar flow, Sensors and Actuators B-Chemical 75 (2001) 213-222.

[11] M. Hill, Y. Shen, J.J. Hawkes, Modelling of layered resonators for ultrasonic separation, Ultrasonics 40 (2002) 385-392.

[12] O. Doblhoffdier, T. Gaida, H. Katinger, W. Burger, M. Groschl, E. Benes, A novel ultrasonic resonance field device for the retention of animal-cells, Biotechnology Progress 10 (1994) 428-432.

[13] H. Bohm, L.G. Briarty, K.C. Lowe, J.B. Power, E. Benes, M.R. Davey, Quantification of a novel h-shaped ultrasonic resonator for separation of biomaterials under terrestrial gravity and microgravity conditions, Biotechnology and Bioengineering 82 (2003) 74-85.

[14] N.R. Harris, M. Hill, S.P. Beeby, Y. Shen, N.M. White, J.J. Hawkes, W.T. Coakley, A silicon microfluidic ultrasonic separator, Sensors and Actuators B 95 (2003) 425-434.
15] J.J. Hawkes, M. Gröschl, H. Nowotny, S. Armstrong, P. Tasker, W.T. Coakley, E. Benes, Single half wavelength ultrasonic particle filter: Predictions of the transfer matrix multi-layer resonator model and experimental filtration results, Journal of the Acoustical Society of America 111 (2002) 1259-1266.

[16] F. Petersson, A. Nilsson, C. Holm, H. Jonsson, T. Laurell, Continuous separation of lipid particles from erythrocytes by means of laminar flow and acoustic standing wave forces, Lab on a Chip 5 (2005) 20-22.

[17] A. Neild, S. Oberti, J. Dual, Design, modeling and characterization of microfluidic devices for ultrasonic manipulation, Sensors and Actuators B: Chemical 121 (2007) 452-461

[18] H. Nowotny, E. Benes, General one-dimensional treatment of the layered piezoelectric resonator with 2 electrodes, Journal of the Acoustical Society of America 82 (1987) 513-521.

[19] M. Hill, R.J.K. Wood, Modelling in the design of a flow-through ultrasonic separator, Ultrasonics 38 (2000) 662-665.

[20] M. Hill, The selection of layer thicknesses to control acoustic radiation force profiles in layered resonators, JASA 114 (2003) 2654-2661.

21] L.P. Gor'kov, On the forces acting on a small particle in an acoustical field in an ideal fluid, Soviet Physics Dokl. 6 (1962) 773-775.

[22] S.P. Martin, R.J. Townsend, L.A. Kuznetsova, K.A.J. Borthwick, M. Hill, M.B. McDonnell, W.T. Coakley, Spore and micro-particle capture on an immunosensor surface in an ultrasound standing wave system, Biosensors and Bioelectronics 21 (2005) 758-767.

[23] R.J. Townsend, M. Hill, N.R. Harris, N.M. White, Modelling of particle paths passing through an ultrasonic standing wave, Ultrasonics 42 (2004) 319324

[24] R.J. Townsend, M. Hill, N. Harris, M.B. McDonnell, Performance of quarterwavelength particle concentrators, Ultrasonics 48 (2008) 515-520.

[25] Onda Corporation, Tables of Acoustic Properties of Materials, <http:// www.ondacorp.com/tecref_acoustictable.html>, 2007 (accessed 03.07).

[26] R.J. Townsend, M. Hill, N.R. Harris, N.M. White, Investigation of twodimensional acoustic resonant modes in a particle separator, Ultrasonics 44 (2006) e467-e471. 